\title{
Correspondence
}

\section{Covesdem syndrome}

SIR,

I refer to our article 'Recessively inherited costovertebral segmentation defect with mesomelia and peculiar facies (Covesdem syndrome). A new genetic entity?' published in the Journal $(15,123-$ $127,1978)$. It has since been brought to our notice (R. Gorlin, 1978, personal communication) that a similar report has been published before by Robinow and Silverman (1969). They described a dominant pedigree with face, genital, and limb abnormalities similar to our cases. Of 4 cases, one patient had a single hemivertebra, and in one 'the lateral ossification centres of T10 vertebrae were separate'. They disregarded these vertebral anomalies, but stressed instead a progressive dwarfism not seen in our cases. Subsequently, reports, usually of sporadic cases, have stressed the vertebral anomalies (Vera Roman, 1973). The syndrome to which the term 'fetal face syndrome' is sometimes applied is described as dominant, whereas ours was clearly recessive. Wadlington et al. (1973) described 2 sibs with normal parents and short grandparents, whom they discussed as possibly recessive or possibly dominant with reduced penetrance. Dr Gorlin also had personal communication from J. Opitz describing 3 sibs of consanguineous parents.

We wish to congratulate Dr Gorlin for spotting the similarity to our report. As we postulated, the combination of costovertebral segmentation defect plus mesomelic dwarfism plus abnormal facies (and abnormal genitalia) does in fact represent a separate. genetic syndrome. A dominant form (the Robinow syndrome) and a recessive form (?Covesden syndrome) clearly exist. Our blind spot is regretteditu

R. S. WADIAT Ruby Hall Clinic N 40 Sassoon Road?

Poona 411001

\section{References} Indiog

Robinow, M., and Silverman, F. N. (1969). A newly recog nised dwarfing syndrome. American Journal of Diseases of Children, 117, 645-651.

Vera Roman, J. M. (1973). Robinow dwarfism accompanie by penile agenesis and hemivertebrae. American Journak of Diseases of Children, 126, 206-208.

Wadlington, W. B., Tucker, V. L., and Schimke, R. N. (1973) Mesomelic dwarfism with hemivertebrae and smalf genitalia (the Robinow syndrome). American Journal o尺 Diseases of Children, 126, 202-205. 